BIO Web of Conferences 2, 05003 (2014)

DOI: $10.1051 /$ bioconf/ 20140205003

(C) Owned by the authors, published by EDP Sciences, 2014

\title{
Development of a new method to identify aminoacylated RNA
}

\author{
Ji Wang ${ }^{1}$, Shixin $\mathrm{Ye}^{2}$, Daniel Gautheret ${ }^{1}$, and Jean Lehmann ${ }^{1}$ \\ ${ }^{1}$ Institut de Génétique et Microbiologie - Université Paris-Sud, F - 91405 Orsay cedex \\ ${ }^{2}$ Institut de Biologie, Section Neurosciences, Ecole Normale Supérieure, 46 rue d’Ulm, F - 75005 \\ Paris
}

\begin{abstract}
A RT-PCR method is developed to isolate RNA aminoacylated on their 3' end from large pools of RNA. The method is being applied in two separate projects. We are interested in isolating a new class of ribozymes that could successively catalyze the two chemical reactions leading to their own 3' aminoacylation (ATP activation of an amino acid followed by 3 ' esterification of the RNA). The catalysis of each of the two reactions has independently been demonstrated for some RNA isolated with the SELEX methodology [1-2]. However, the coupling of both reactions on a same molecule has not been achieved yet. The identification of these still hypothetical ribozymes may help understand how the former translation system started in the absence of the aminoacyltRNA Synthetase, which catalyzes the above two reactions on tRNA in modern cells. In another project, we would like to identify the whole repertoire of aminoacylated RNA (the "aminoacylome") in cells. There are strong indications that other RNA besides tRNA and tmRNA may be aminoacylated for biological purposes [3-4].
\end{abstract}

\section{Summary of the methodology}

We are developing a RT-PCR method to isolate RNA aminoacylated on their 3' end from large pools of RNA. The method relies on the use of sodium or potassium periodate to oxidize the 3' end of RNA that are unprotected by an amino acid. Subsequent to periodate treatment, the amino acids are being removed from the 3' end while increasing the $\mathrm{pH}$, making the 3' end of these RNA available for RNA ligation. An RNA adaptor is ligated to unoxidized RNA with a T4 RNA ligase, which allows their reverse transcription from a primer complementary to the adaptor, generating a cDNA. A DNA duplex is subsequently ligated at the 3' end of the cDNA with a T4 DNA ligase to allow PCR amplification. Sequences that are included into the final construction are identified with the high throughput sequencing technology (Illumina).

This method is now being used in two separate project:

1) As part of our investigations on the origin of the genetic code, we would like to identify a new class of ribozyme that could successively activate an amino acid with ATP and transfer the amino acid onto its own 3' end. Such hypothetical RNA could play the role of tRNA at the origin of Life in the absence of aminoacyl-tRNA synthetase.

2) In line with our interest in regulatory RNA, we believe that this methodology may uncover 
RNA in modern cells that are aminoacylated for regulatory purposes. The method will be applied on total RNA from cellular extracts. So far, only tRNA and mtRNA are known to undergo aminoacylation.

\section{Advantages of the method compared to existing protocols}

The method should allow the identification of any aminoacylated RNA molecule without the requirement of constant (known) regions at the 3' end 5' end. These regions are necessary for PCR amplification. In the above method, they are added during the isolation procedure. The pools of RNA used in earlier SELEX studies on ribozymes [1-2] were indeed not entirely random due to the presence of these constant regions, which can impose prohibitive constraints on catalysis [5], and thus on the kind of ribozmes that could be isolated. In both projects, the selection step is extremely sharp since the high concentration of periodate used in the procedure (of the order of $50 \mathrm{mM}$ ) may completely wipe out from the isolation procedure RNA the 3' end of which are not protected by an amino acid.

\section{References}

[1]. Illangasekare, M., Sanchez, G., Nickles, T., Yarus, M. Aminoacyl-RNA Synthesis Catalyzed by an RNA. Science 267, 643-647 (1995)

[2]. Kumar, R. K. \& Yarus, M. RNA-catalyzed amino acid activation. Biochemistry 40, 6998-7004 (2001)

[3]. Francklyn C, Schimmel, P. Enzymatic aminoacylation of an eight-base-pair microhelix with histidine. Proc Natl Acad Sci U S A. 87: 8655-8659 (1990)

[4]. Dreher, T. W. Role of tRNA-like structures in controlling plant virus replication. Virus Res. 139: 217-229 (2009)

[5]. Lehmann, J. Reichel, A., Buguin, A, Libchaber, A. Efficiency of a self-aminoacylating ribozyme: effect of the length and base-composition of its 3' extension. RNA 13: 1191-1197 (2007) 